\title{
Distance Cues and Fields of View in Rear Vision Systems
}

Michael J. Flannagan and Michael Sivak

The University of Michigan

Reprinted From: Automotive Lighting Technology and Human Factors in Driver Vision and Lighting; Rear Vision and Indirect Vision

(SP-1993)

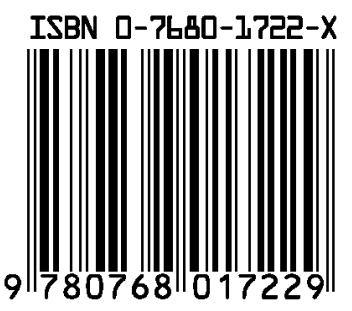


The Engineering Meetings Board has approved this paper for publication. It has successfully completed SAE's peer review process under the supervision of the session organizer. This process requires a minimum of three (3) reviews by industry experts.

All rights reserved. No part of this publication may be reproduced, stored in a retrieval system, or transmitted, in any form or by any means, electronic, mechanical, photocopying, recording, or otherwise, without the prior written permission of SAE.

For permission and licensing requests contact:

SAE Permissions
400 Commonwealth Drive
Warrendale, PA 15096-0001-USA
Email: permissions@ @ sae.org
Tel: $\quad 724-772-4028$
Fax: $\quad 724-776-3036$

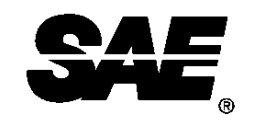

Global Mobility Database ${ }^{\circledast}$

All SAE papers, standards, and selected books are abstracted and indexed in the Global Mobility Database.

For multiple print copies contact:

SAE Customer Service

Tel: $\quad$ 877-606-7323 (inside USA and Canada)

Tel: $\quad$ 724-776-4970 (outside USA)

Fax: $\quad 724-776-0790$

Email: CustomerService@sae.org

\section{ISSN 0148-7191}

\section{Copyright $\odot 2006$ SAE International}

Positions and opinions advanced in this paper are those of the author(s) and not necessarily those of SAE. The author is solely responsible for the content of the paper. A process is available by which discussions will be printed with the paper if it is published in SAE Transactions.

Persons wishing to submit papers to be considered for presentation or publication by SAE should send the manuscript or a 300 word abstract to Secretary, Engineering Meetings Board, SAE. 


\title{
Distance Cues and Fields of View in Rear Vision Systems
}

\author{
Michael J. Flannagan and Michael Sivak \\ The University of Michigan
}

Copyright $@ 2006$ SAE International

\begin{abstract}
The effects of image size on perceived distance have been of concern for convex rearview mirrors as well as camera-based rear vision systems. We suggest that the importance of image size is limited to cases-such as current rearview mirrors - in which the field of view is small. With larger, richer fields of view it is likely that other distance cues will dominate image size, thereby substantially diminishing the concern that distortions of size will result in distortions of distance perception. We report results from an experiment performed in a driving simulator, with static simulated rearward images, in which subjects were asked to make judgments about the distance to a rearward vehicle. The images showed a field of view substantially wider than provided by any of the individual rearview mirrors in current systems. The field of view was 38 degrees wide and was presented on displays that were either 16.7 or 8.5 degrees wide, thus minifying images by factors of 0.44 or 0.22 . In contrast to previous studies of convex mirrors and camera-based rear vision systems, judgments about distance were not affected by image size, suggesting that distance cues other than size were available to subjects as they made their judgments. Further testing is needed to better understand the nature of the distance cues that drivers are able to use in camera-based rear vision systems, but these results suggest that, at least under some conditions, image size is not critical for reliable perception of distance.
\end{abstract}

\section{INTRODUCTION}

The small size of images in convex rearview mirrors results in overestimation of the distances to rearward vehicles-a circumstance that has generated much concern and motivated many studies over the years. There has been similar concern about how the effective magnification of camera-based rear-vision systems may affect perception of distance. We have argued in the past that the effect of magnification on distance perception is unusually strong in the case of rearview mirrors because of the limited fields of view that they provide (Flannagan \& Sivak, 2005). With larger fields of view, and the richer distance cues that those fields are likely to provide, the importance of magnification might be greatly diminished. In the present study, we investigated the perception of distance in images of rearward scenes with relatively large fields of view at two very different levels of magnification.

The rearview mirror systems on current passenger cars involve three separate mirrors, each of which shows only a small portion of the rearward scene. As they are actually mounted and aimed, the typical horizontal extents of the fields of view are 12.9 degrees for left exterior mirrors, 25.3 degrees for center mirrors, and 22.5 degrees for right exterior mirrors (Reed, Lehto, \& Flannagan, 2000). It may be that the small sizes of these fields limit the usefulness of a variety of distance cues that could be used more effectively in a rear vision system with a larger, continuous field of view (Flannagan \& Sivak, 2005).

In fact, it could be argued that the restricted fields provided by current rearview mirrors are similar to the experimental conditions used by Holway and Boring (1941) in their classic study of how perceptions of distance and size are affected by progressively removing distance cues. In their final, most minimal condition, subjects viewed an isolated target in a narrow, featureless tube that was designed to eliminate, as much as possible, the distance cues provided by the visual context around the target. In that condition, visual angle was virtually the only distance cue remaining, and, not surprisingly, that cue dominated subjects' judgments. The image of a rearward vehicle in a typical passengerside rearview mirror is often nearly as isolated, and it is therefore perhaps not surprising that, under those circumstances, image minification can make vehicles appear farther away. 
The results of numerous studies indicate that, while several factors can diminish the effect of mirror convexity and image size on distance perception, the effect is almost always present to some extent (Flannagan, 2000). Previous studies of camera-based systems have also shown effects of magnification. For example, we performed a study in fully dynamic driving conditions using a task that required subjects to judge the last possible time at which they could change lanes in front of an overtaking car that was seen in a rear-vision system (Flannagan \& Mefford, 2005). A change in magnification by a factor of 0.50 (from unit magnification to half that) caused a significant reduction in the distance at which a lane change was judged to be safe (35.4 meters at unit magnification, versus 27.5 meters at 0.50 magnification). The reduction in distance was less than proportional to the change in magnification $(27.5 / 35.4=$ 0.78 , versus 0.50 ), but still substantial.

The issue of what magnification should be used for camera-based rear vision systems is not yet settled. Candidates vary: a factor of 1.25 was proposed by Roscoe (1984) for a wide range of systems that would include automotive camera systems; 0.33 was suggested by Hicks et al. (1999); and the federal standard for rearview mirrors (FMVSS 111) requires that driver-side and interior mirrors be unit magnification, a requirement that, if extended to rear vision systems in general, would imply a factor of 1.00 . Complicating matters further, the effective magnification of any rearvision system that uses a video screen will depend on the eye-to-screen distance (Flannagan, Sivak, \& Mefford, 2002). Given the ambiguity about the role of image size and magnification, it may be especially worthwhile to evaluate the conditions under which these variables may or may not be critical.

Our objective in the current study was to assess subjects' perception of the distance to a rearward vehicle when they were presented with a field of view substantially wider than that provided by current rearview mirrors. We were limited to some extent by practical considerations of screen size and resolution, and we therefore decided to present only one half of the rearward scene (the right side). This allowed us to present our larger magnification level on a screen that was modest in size $(24.0 \mathrm{~cm}$ wide), although still very large relative to screens currently in use on vehicles. In a practical rear vision system, we would expect that presenting an even more extensive rear scene would be desirable.

In order to control the availability of distance cues, we used static, simulated rear scenes, and presented them to subjects in a driving simulator. In each rear scene, a vehicle was visible at some distance to the rear and one lane to the right. In order to assess the possible role of different vantage points and the visibility of parts of one's own vehicle, we used images corresponding to two vantage points: the interior and right exterior rearview mirrors.

We presented exactly the same images at two levels of magnification. Both magnification levels were small, in order to allow a reasonably wide field of view, but they were markedly different from each other in order to make it more likely to observe an effect of magnification if one existed. As viewed by the subjects, the magnification levels were 0.22 and 0.44 , where 1.0 would correspond to a situation in which the images spanned the same visual angles as the real scenes viewed directly.

\section{METHOD}

\section{SUBJECTS}

Fifteen licensed, active drivers participated in the experiment as paid subjects. There were seven in a younger group (from 25 to 30 years old, with an average age of 27.9), and eight in an older group (61 to 80, mean 69.4). The younger group consisted of four males and three females, and the older group consisted of four males and four females. Visual acuity for the entire group ranged from $20 / 13$ to $20 / 35$ with a mean of $20 / 22$.

\section{EQUIPMENT}

The experiment was performed in the UMTRI driving simulator. The subject's position and the general configuration of the simulator are illustrated in Figure 1. The forward screens of the simulator were not actually involved in the subject's task, but, in order to be compatible with the images shown on the rear vision display, they showed a featureless gray ground surface below a featureless blue sky. The subject was not required to drive during the study; the simulator was used only to provide a visual environment similar to an actual vehicle.

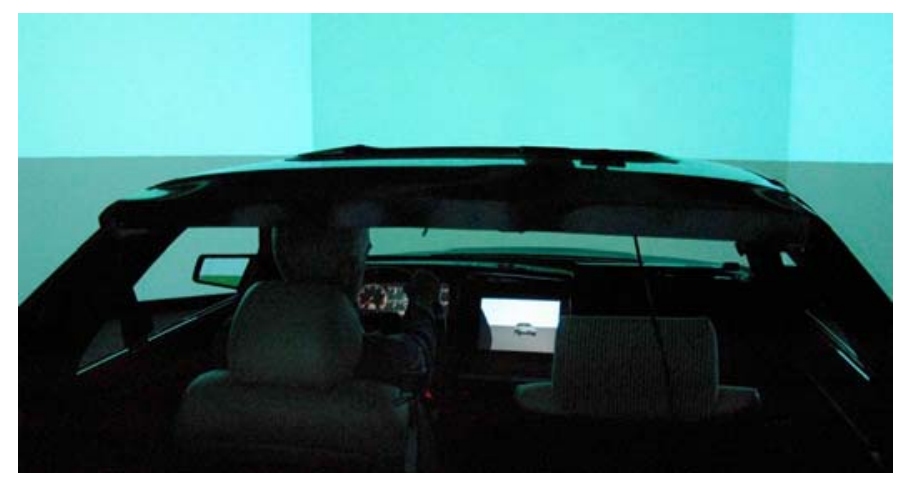

Figure 1. The driving simulator, seen from above and behind the subject's position, showing part of the front screen, and the rear vision display (at the larger of the two magnification levels) in the middle of the instrument panel. 
Simulated camera rear views were presented on a color LCD screen in the middle of the instrument panel, as shown in Figure 1. The screen was surrounded by a simple black mask with a rectangular opening either 24.0 $\mathrm{cm}$ wide by $16.0 \mathrm{~cm}$ high (for the higher magnification level) or $12.0 \mathrm{~cm}$ wide by $8.0 \mathrm{~cm}$ high (for the lower magnification level). The simulated rear scenes were always scaled to these mask sizes, so that the scenes were always framed the same way and contained the same images, at the larger and smaller sizes. The rearward scene shown in Figure 1 is at the higher of the two magnification levels used. Screen resolution was 4.2 pixels $/ \mathrm{mm}$.

The display screen used for the rearward images was about $80 \mathrm{~cm}$ from a typical subject's eye position. The location of subjects' eyes was controlled to some degree by keeping the driver's seat in the same position for all subjects, but small variations between subjects, and within individual subjects' sessions because of slight shifts in position, were allowed. The resulting horizontal visual angles were about 16.7 and 8.5 degrees for the larger and smaller magnifications, respectively.

\section{STIMULI}

Photographs of a 1993 Nissan Altima taken in a real roadway setting were used to produce the rearward views. The Altima was present in each photograph, at distances varying from 4 to $20 \mathrm{~m}$ in $1-\mathrm{m}$ increments. Distance was measured from the camera position to the front of the Altima. The photographs were taken from positions in a lane one lane to the left of the Altima.

Two vantage points were used: one corresponding to the inside rearview mirror of a typical passenger car and one corresponding to the right outside rearview mirror of a typical passenger car. The images were taken from those vantage points without an actual observer car being present. A camera on a tripod was placed on the pavement at the appropriate height, lateral location in the lane, and longitudinal distance from the rearward car. The vantage point for the center mirror was $119 \mathrm{~cm}$ high and at the middle of the observer-car lane. The vantage point corresponding to the right exterior mirror was $95 \mathrm{~cm}$ high and $87 \mathrm{~cm}$ to the right of the center of the observer-car lane. These distances were based on average locations of rearview mirrors on passenger cars, as measured by Reed, Lehto, and Flannagan (2000).

Images were taken with a Nikon D70 digital camera, using a Nikkor $18-70 \mathrm{~mm}$ zoom lens. The focal length was set to $35 \mathrm{~mm}$, providing a field of view of about 38 degrees. Figure 2 shows schematically the approximate fields of view for the two vantage points in relation to a typical passenger car. The images were taken on an overcast day so that the lighting of the vehicle would be uniform, with minimal shadows or highlights on the vehicle surfaces.

The images of the Altima were isolated from the background of the real road on which they were taken and superimposed on a very simple background that was the same as the scene presented on the forward screens of the simulator, i.e., a simple virtual world consisting of a featureless gray ground surface below a featureless blue sky. This eliminated all distance cues, such as converging lane lines, that were not part of the image of the Altima itself or based on its horizontal or vertical location in the visual field.

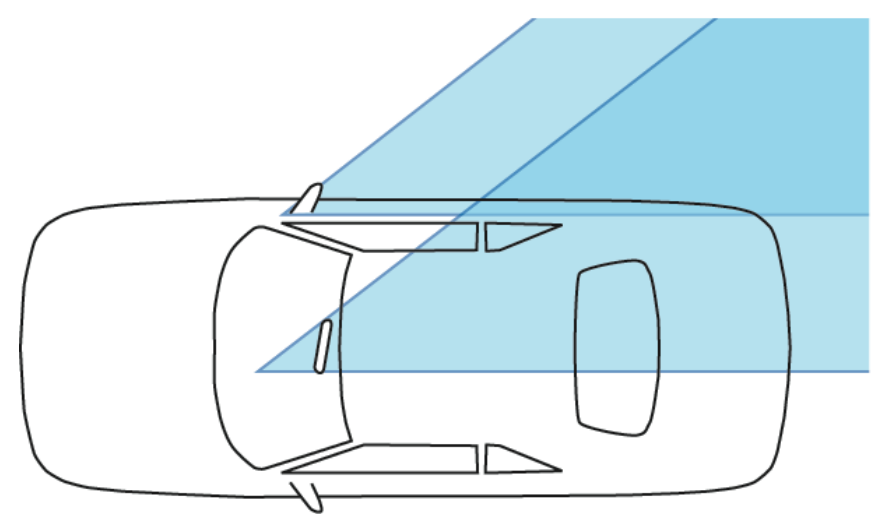

Figure 2. Schematic depiction of the exterior and interior vantage points and fields of view represented in the rearward images.

Silhouettes of the interior or rear side panel of a typical sedan (in fact, the same 1993 Nissan Altima that was used as the rearward car) were superimposed on the images of the rearward scene. The interior was superimposed on the images taken from the vantage point of the interior mirror, and the side panel was superimposed on the images taken from the vantage point of the exterior mirror.

The simulated images always corresponded to at least a small gap between the front of the rearward vehicle and the rear of the forward vehicle. (This assumes that the forward vehicle, from which the simulated scene is supposedly being observed, is the size of a typical passenger car.) At the closest simulated distance, the rearward vehicle was $4 \mathrm{~m}$ behind the scene's vantage point, which corresponded to either the interior or right exterior mirror. On typical passenger cars, and with typical seating positions, those vantage points are about $3.5 \mathrm{~m}$ forward of the rear of the vehicle. Therefore, roughly speaking, in the closest situations the clearance specified by the simulated images was about $0.5 \mathrm{~m}$. For the furthest situations, those with a nominal distance of $20 \mathrm{~m}$, the clearance was about: $20-3.5=16.5 \mathrm{~m}$.

Figure 3 shows examples of the resulting images, at several distances, for the exterior and interior vantage 
points. All images were mirror reversed so that they would have the same spatial layout as images seen in rearview mirrors.

The same images were used for the large and small magnification conditions. They were presented at 24.0 by $16.0 \mathrm{~cm}$ for the larger magnification condition, and 12.0 by $8.0 \mathrm{~cm}$ for the smaller magnification condition. As indicated above, at the viewing distance of about $80.0 \mathrm{~cm}$, the horizontal dimensions of the stimuli corresponded to 16.7 and 8.5 degrees. Because the horizontal extent of the actual field of view was 38 degrees, the resulting magnification factors were 0.44 and 0.22 , respectively.

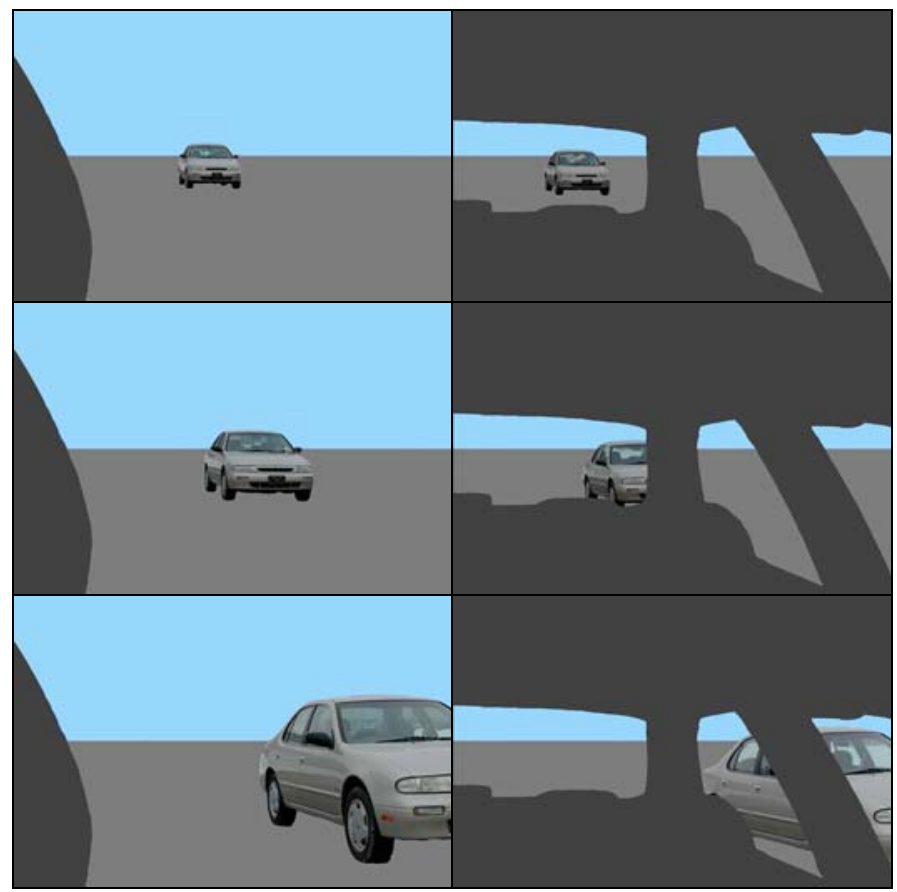

Figure 3. Examples of the images presented to subjects, from the exterior (left column) and interior (right column) vantage points; at the furthest (20 m, top row), middle (12 $\mathrm{m}$, middle row), and nearest $(4 \mathrm{~m}$, bottom row) distances. These are mirror-reversed views of a vehicle one lane to the right.

\section{PROCEDURE}

Subjects participated in individual sessions of about one hour each. After receiving instructions, they viewed a series of images on the rear vision display. The images were presented for 1 second each, and the subjects were instructed to think of the images as representing brief views of a dynamic traffic situation in which they and the vehicle behind them were traveling at the same speed, and thus maintaining the same relative position.

After viewing each image, the subjects said "yes" or "no" to indicate whether or not they believed there was enough clearance between themselves and the rearward vehicle to allow them to safely change lanes in front of it.
The size of an acceptable gap was left entirely to the subjects' judgment; they were merely instructed to be as consistent as possible in their responses. (Objectively, as described above, all of the simulated images implied at least a small gap between the front of the rearward vehicle and the rear of the vehicle that the subject was supposed to be sitting in, assuming the latter vehicle to be the size of a typical passenger car.)

For each subject, the magnification of the rearward scenes was the same throughout the session: either 0.44 or 0.22 times the visual angle of the real scene as viewed from the relevant vantage point. Eight subjects (four in the older group and four in the younger group) saw the 0.44 magnification, and seven (four in the older group and three in the younger group) saw the 0.22 magnification.

Each subject saw four blocks of trials. The vantage point (exterior or interior) was the same throughout each block and was changed between blocks. The order of presentation of the vantage points was balanced across subjects. In each block, each of the 17 distances from 4 to $20 \mathrm{~m}$ was presented once, in random order.

\section{RESULTS}

As expected, the proportion of trials on which subjects judged that a lane change was possible increased with distance. The overall result is shown in Figure 4. The distance at which half the judgments were "yes" was about $11.8 \mathrm{~m}$, measured from the vantage point to the front bumper of the rearward vehicle. If we assume, as is reasonable for mirrors on a typical passenger car, that the vantage point is about $3.5 \mathrm{~m}$ ahead of the rear of the observing driver's own car, then this result corresponds to a gap of about $11.8-3.5=8.3 \mathrm{~m}$ between the rear bumper of the forward car and the front bumper of the rearward car.

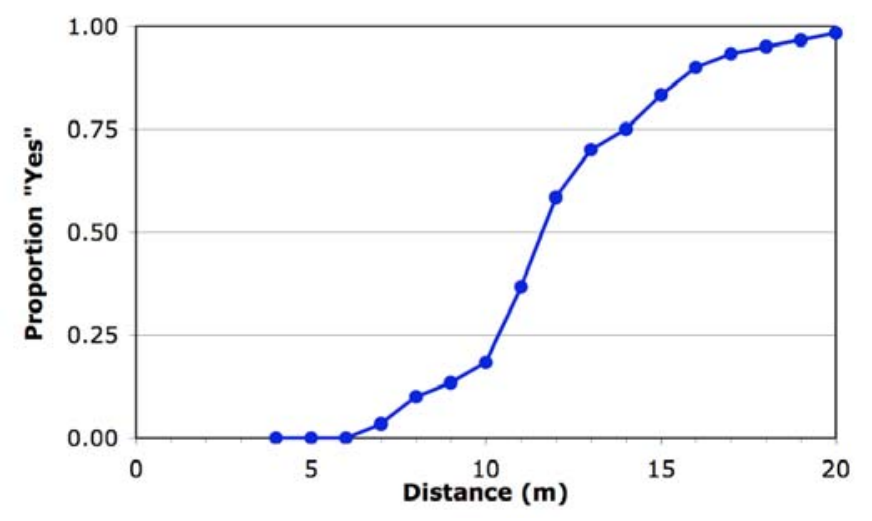

Figure 4. Proportion of "yes" judgments, indicating that a lane change was possible, as a function of distance to the rearward vehicle, summarized over all subjects and conditions. 
The shortest distance at which there were any "yes" judgments was $7 \mathrm{~m}$. Making the same adjustment of 3.5 $\mathrm{m}$, this corresponds to a gap of about $7.0-3.5=3.5 \mathrm{~m}$ from bumper to bumper. Although the experimental task that we used differed from real driving in several ways, it is interesting to compare this value to the estimate of minimum gap in real lane changes made by Smith, Glassco, Chang, and Cohen (2003). They used data from a study in which the normal lane-change behavior of drivers in real traffic could be measured with a sensor mounted on an instrumented vehicle. Although other issues were involved in the study, for this purpose the instrumented vehicle functioned simply as an observation platform and was not itself substantially involved in the maneuvers of interest.

The first thing to note about the data from Smith et al. is that, in the real maneuvers they observed, when a vehicle changed lanes in front of another vehicle, the vehicle that was changing lanes was usually moving faster than the other vehicle. Our simulated conditions are therefore somewhat atypical in that we represented the traffic scenario to subjects as one in which they and the other vehicle were going at the same speed. Furthermore, the sample of maneuvers measured by Smith et al. do not necessarily represent all real world conditions. However, it still may be useful to compare the size of gaps accepted. Smith et al. found that, when a lane-changing vehicle first began to move laterally, the longitudinal gap to a vehicle in the adjacent lane varied with the relative speed of the two vehicles and the gap was sometimes negative (i.e., there was still longitudinal overlap between the two vehicles at the beginning of the lateral movement). They also measured the longitudinal gaps when the lane-changing vehicles had moved far enough to overlap laterally with the vehicles in the lane they were entering. Independent of relative speed, the minimum gap was about $4 \mathrm{~m}$ (from the rear bumper of the vehicle changing lanes to the front bumper of the other vehicle). That value is very similar to the corresponding gap of $3.5 \mathrm{~m}$ in our data, suggesting that our subjects may have been responding to the simulated visual conditions in a way similar to how they would respond in actual driving. The relevant images (for distances from the vantage point to the rear car of $7 \mathrm{~m}$ ) are shown in Figure 5.

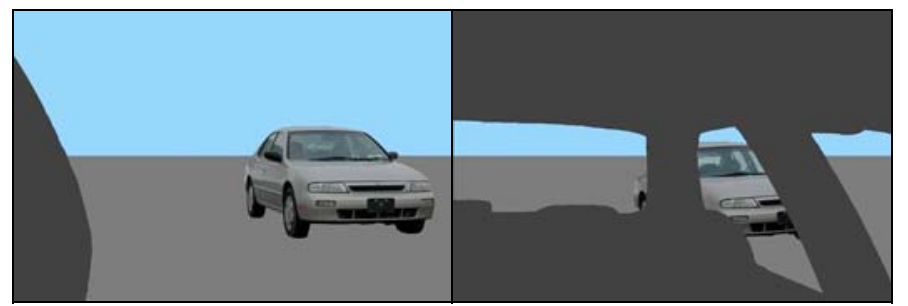

Figure 5. Images from the exterior (left) and interior (right) vantage points, for the shortest distance at which there were any judgments that a lane change could be made $(7 \mathrm{~m})$.
The same judgments as in Figure 4, broken down by the two magnification levels, are shown in Figure 6. The functions are roughly the same for the two conditions. There is some indication that the transition from 0 to 1 in proportion of "yes" responses took place over a wider range of distance in the 0.22 magnification condition than in the 0.44 magnification condition. However, the functions in Figure 6 show the combined results of all subjects, and so, although they serve to describe the general pattern of results, they are somewhat difficult to interpret. Specifically, the transitions from 0 to 1 in Figure 6 are caused by a combination of inconsistency within individuals and across individuals in judging whether any given distance would permit a lane change. In the results of a logistic analysis, reported below, there is no statistically significant effect of magnification on the rates of transition between 0 and 1 .

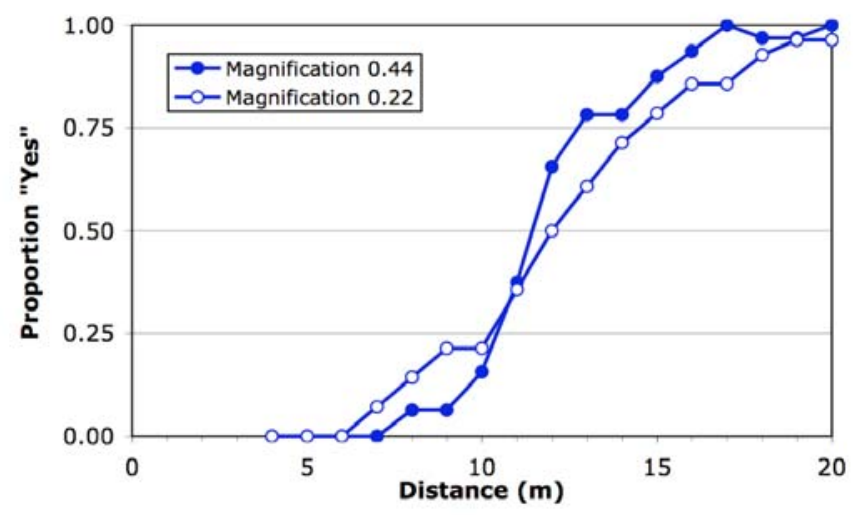

Figure 6. Proportion of "yes" judgments as functions of distance to the rearward vehicle for the two levels of magnification.

Judgments broken down by the two vantage points are shown in Figure 7 . In contrast to the breakdown by magnification, here there seems to be a reasonably clear difference, in which subjects accepted shorter distances with the exterior view. However, the logistic analysis provides a clearer analysis of this effect as well.

To provide a fuller analysis of the data, including the consistency of effects over subjects, we fit models to each subject's data individually, using logistic models to describe the rise in proportion of "yes" responses as shown in Figures 4, 6, and 7. Estimates of the distances at which each individual subject's tendency to say "yes" reached a proportion of 0.50 were calculated, and the results were analyzed with mixed models analysis of variance. 


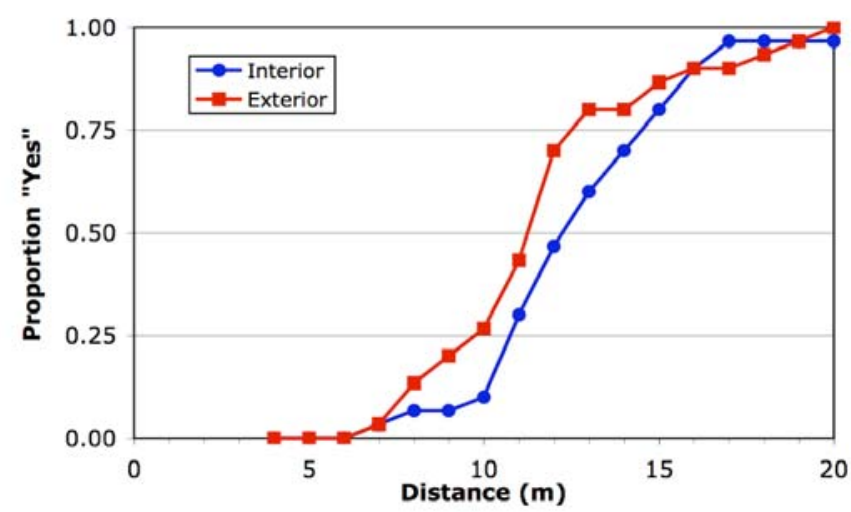

Figure 7. Proportion of "yes" judgments as functions of distance to the rearward vehicle for the two vantage points.

The effect of age group was significant, $F(1,12)=5.33, p$ $<.05$, as was the effect of vantage point, $F(1,12)=6.88$, $p<.05$. As might be expected from the pattern in Figure 6 , the effect of magnification was not significant, $F(1,12)=0.02$.

Over all conditions, the distance at which the proportion of "yes" judgments reached 0.50 was $11.8 \mathrm{~m}$. The corresponding distance in the condition with the exterior vantage point was slightly shorter $(11.6 \mathrm{~m})$ than with the interior vantage point $(12.1 \mathrm{~m})$. Younger subjects judged lane changes to be possible at shorter distances (10.3 $\mathrm{m})$ than older subjects $(13.2 \mathrm{~m})$.

Figure 8 shows mean distances for each combination of subject age group and vantage point. The effect of age is considerably larger than the effect of vantage point.

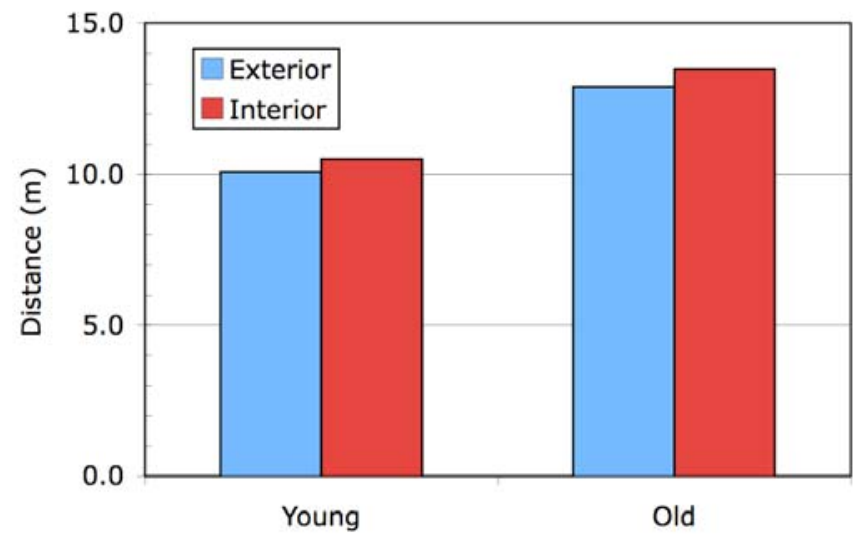

Age Group

Figure 8. Distances at which responses were half "yes" for each combination of age group and vantage point, derived from logistic models fit for individual subjects.
The effect of vantage point is too small to be of much practical significance, but it is intriguing. One possible interpretation is that there is a tendency for distances to look greater in the exterior condition because of the relative lack of a frame of reference in comparison to the strong frame of reference offered by the window openings and pillars in the internal condition. Perhaps with somewhat weaker reference points the levels of image minification used in this experiment $(0.44$ or 0.22$)$ were able to have some small effect on distance perception.

\section{DISCUSSION AND CONCLUSIONS}

In contrast to previous studies of distance perception in convex mirrors and camera-based rear vision systems, in the present experiment image magnification had no effect. This is particularly interesting because the difference in magnification that was used here was reasonably strong $-a$ factor of 2 . It seems likely that the reason for this is that, with the large fields of view used here, subjects had enough cues other than image size to use in making their judgments. Quite possibly, lateral location in the field of view, particularly in relationship to the framework provided by parts of the observer car, was one such cue. The slight difference in results between the interior vantage point (which involved a very rich framework) and the exterior vantage point (which involved a relatively limited framework) offers some support for this interpretation.

The fact that even a strong difference in magnification does not have a measurable effect on distance judgments suggests that magnification may not be a very critical variable for certain types of camera-based rear vision systems-specifically, those that involve reasonably extensive, continuous fields of view. The images used in the present experiment provided reasonably wide fields of view (38 degrees), but it would probably be good to provide even wider fields. The ideal may be a single, extremely wide display that provides a unified view of the entire rearward scene (e.g., Flannagan et al., 2005; Hicks et al., 1999).

We had expected that there would be a more substantial effect of vantage point, with distance perception being more reliable with the internal vantage point because of the richer set of landmarks provided by the view of the interior of the car. However, it appears that the location in the field of view, or perhaps other details of the appearance of the rearward car, were useful enough as distance cues to make distance perception reliable for both vantage points.

The stimuli used here were artificial and limited in various ways. For the most part, we expect that more realistic conditions would provide even more alternative distance cues (e.g., converging lane lines, road surface 
texture gradients, relative size of roadside objects), and that the importance of magnification should therefore be even less than in the present results. However, there are likely exceptions to this, especially night conditions, in which perception is generally difficult. Further work should examine distance perception under a wider range of conditions than were used in this study.

In this study, although we presented subjects with a substantially wider field of view than current mirrors provide, it was still less than half of what is probably needed for a reasonably complete rearward view. Partly, this was because we did not want to use extremely minified images. Given that the minification levels used here (0.44 and 0.22) did not affect distance perception, it would be desirable to test the effects of even smaller image sizes. However, for smaller images there are concerns other than distance perception that may become dominant, including the resolution limits of either the display or the driver's own visual capabilities (e.g., Hicks et al., 1999).

\section{ACKNOWLEDGMENTS}

We wish to thank Ichikoh Industries, Ltd. for their generous support of this research.

\section{REFERENCES}

1. Flannagan, M. J. (2000). Current status and future prospects for nonplanar rearview mirrors (SAE Technical Paper Series No. 2000-01-0324). Warrendale, Pennsylvania: Society of Automotive Engineers.

2. Flannagan, M. J., \& Mefford, M. L. (2005). Distance perception with a camera-based rear vision system in actual driving (Report No. UMTRI-2005-38). Ann Arbor: The University of Michigan Transportation Research Institute.

3. Flannagan, M. J., \& Sivak, M. (2005). Driver workload for rear-vision systems with single versus multiple display locations (SAE Technical Paper Series No. 2005-01-0445). Warrendale, Pennsylvania: Society of Automotive Engineers.

4. Flannagan, M. J., Sivak, M., \& Mefford, M. L. (2002). Distance perception in camera-based rear vision systems (SAE Technical Paper Series No. 2002-010012). Warrendale, Pennsylvania: Society of Automotive Engineers.

5. Hicks, R., Schofield, K., Tarnow, P., \& Veiseh, M. (1999). Panoramic electronic rear vision for automotive applications (SAE Technical Paper Series No. 1999-01-0655). Warrendale, Pennsylvania: Society of Automotive Engineers.
6. Holway, A. H., \& Boring, E. G. (1941). Determinants of apparent visual size with distance variant. The American Journal of Psychology, 54(1), 21-37.

7. Reed, M. P., Lehto, M. M., \& Flannagan, M. J. (2000). Field of view in passenger car mirrors (Report No. UMTRI-2000-23). Ann Arbor: The University of Michigan Transportation Research Institute.

8. Roscoe, S. N. (1984). Judgments of size and distance with imaging displays. Human Factors, 26(6), 617-629.

9. Smith, D. L., Glassco, R., Chang, J., \& Cohen, D. (2003). Feasibility of modeling lane-change performance (SAE Technical Paper Series No. 2003-01-0280). Warrendale, Pennsylvania: Society of Automotive Engineers. 